\title{
Rate for laser-induced nuclear dipole absorption
}

\author{
Adriana Pálffy, ${ }^{1, *}$ Paul-Gerhard Reinhard $\odot,{ }^{2, \dagger}$ and Hans A. Weidenmüller ${ }^{1, \ddagger}$ \\ ${ }^{1}$ Max-Planck-Institut für Kernphysik, Saupfercheckweg 1, D-69117 Heidelberg, Germany \\ ${ }^{2}$ Institute for Theoretical Physics II, University of Erlangen-Nuremberg, Staudtstrasse 7, 91058 Erlangen, Germany
}

(Received 12 December 2019; revised manuscript received 13 February 2020; accepted 6 March 2020; published 30 March 2020)

\begin{abstract}
Using the Brink-Axel hypothesis we derive the rate $R$ for nuclear dipole excitation by a laser pulse carrying $N \gg 1$ photons with average energy $\hbar \omega_{0} \approx 5 \mathrm{MeV}$. As expected $R \propto\left(\hbar \omega_{0}\right)^{3}$. The rate is also proportional to the aperture $\alpha$ of the laser pulse. Perhaps less expected is the fact that $R \propto N$, irrespective of the degree of coherence of the laser pulse. The expression for $R$, derived for a nearly stationary laser pulse, is valid also for short times and can, thus, be used in simulations via rate equations of multiple nuclear dipole excitations by a single pulse. The explicit dependence of $R$ on the parameters of the laser pulse and on nuclear parameters given in the paper should help to optimize experiments on laser-nucleus reactions.
\end{abstract}

DOI: 10.1103/PhysRevC.101.034619

\section{PURPOSE}

This paper is triggered by recent experimental, computational, and theoretical advances in the production of highenergy laser pulses. Intense pulses with photon energy $\hbar \omega_{0}$ in the 5-MeV range and with a typical energy spread $\sigma$ in the $10-\mathrm{keV}$ range are expected to become available in the near future. Efforts are presently undertaken in this direction at the Nuclear Pillar of the Extreme Light Infrastructure under construction in Romania [1] and in the development of socalled Gamma Factories at the Large Hadron Collider of CERN [2]. For the theoretical description of nuclear reactions induced by such pulses, the use of rate equations is called for. One of the input parameters needed is the rate $R$ for laser-induced nuclear dipole absorption. In previous works [3-5] a plausible guess for the value of $R$ was used. Here we derive an expression for $R$ which displays the dependence of $R$ on the parameters characterizing the laser pulse and the target nucleus. These are, for the laser pulse, in addition to $\hbar \omega_{0}$ and $\sigma$, the total number $N$ of photons in the pulse, and the opening angle $\alpha$ of the aperture of the pulse. For the target nucleus we use the Brink-Axel hypothesis. The Giant Dipole Resonance (GDR) built upon every state of the target nucleus is then characterized by the mean energy $E_{d}$ and by the spreading width $\Gamma^{\downarrow}$. The dependence of $R$ on these

\footnotetext{
*palffy@mpi-hd.mpg.de

${ }^{\dagger}$ paul-gerhard.reinhard@fau.de

†haw@mpi-hd.mpg.de
}

Published by the American Physical Society under the terms of the Creative Commons Attribution 4.0 International license. Further distribution of this work must maintain attribution to the author(s) and the published article's title, journal citation, and DOI. Open access publication funded by the Max Planck Society. parameters (essential for optimizing future experiments) basically confirms previous estimates [4,5]. The rate is boosted by the factor $N$. We show that coherence of the laser pulse is not a necessary requirement for that boost. We compare our approach based on rate equations and valid for nuclear targets with the standard approach to laser-atom interactions that uses the electrical field strength.

Knowledge of the rate is important also in another respect. It allows us to specify the conditions on the laser pulse (and thereby on the mode of its production) that must be fulfilled to guarantee significant nuclear excitation. As explained below in Sec. II, one of the basic mechanisms for the production of a high-energy laser pulse is Compton backscattering of a standard laser pulse on a "flying mirror" of electrons. We emphasize that coherent Compton backscattering is not a requirement. And it suffices that $N \approx 10^{8}$ photons are backscattered, out of a total of perhaps $\approx 10^{11}$ or more [6] in the primary pulse. The resulting conditions on the flying mirror seem realistic.

We explore the dependence of the rate on photon energy, on photon number, and on coherence properties of the laser pulse. We do not address details such as the actual aperture of the laser pulse or the precise form of the GDR for spherical versus deformed nuclei. Addressing such aspects (which will surely become important eventually for the analysis of data) would be premature since until now, the Brink-Axel hypothesis has not been confirmed for nuclear states far above the yrast line. A more precise estimate of the rate will be called for only after our approach has been confirmed semiquantitatively by data.

The paper is structured as follows. Section II describes the physical background and introduces the physical picture used in our approach. In Sec. III we define the interaction Hamiltonian, and we give the expression for the total dipole transition probability and for the rate. The laser-nucleus interaction is not a standard topic in nuclear physics. Therefore, our presentation is rather explicit. The transition rate for dipole 
absorption is calculated stepwise. In Sec. IV we calculate the transition probability in photon space in the long-time limit. The full transition probability (including the nuclear dipole transition) is worked out in Sec. V. In the following two sections the transition probability is converted into a rate by summing over final states. In Sec. VI we perform the sum over final nuclear states, assuming that dipole excitation at energies in the $\mathrm{MeV}$ range occurs predominantly via the GDR. The sum over final photon states is performed in Sec. VII. Our result for the rate is discussed and physically interpreted in Sec. VIII. The implications of the quasistationary approximation for the laser pulse are investigated in Sec. IX. In Sec. X we address the question of whether coherence of the laser pulse is necessary for obtaining the boost factor $N$. The long-time limit used in Sec. IV to calculate $R$ is not obviously appropriate if $R$ is used in rate equations. In Sec. XI we show that the expression for $R$ applies essentially also in that case. In Sec. XII we briefly address the difference between the lasernucleus interaction based on nuclear equilibration treated here and the standard approach to the laser-atom interaction where equilibration typically does not play a role.

\section{BACKGROUND AND PHYSICAL PICTURE}

One of the possible production mechanisms for intense high-energy laser pulses uses the concept of a relativistic flying mirror whose original idea goes back to Einstein [7]. A first intense infrared laser pulse ejects electrons from a nanometer-thin carbon foil. The electrons attain relativistic energies and form a "flying mirror." On that mirror, a second laser pulse is Compton backscattered [6,8-14]. That increases both the energy and the energy spread of the photons in the second pulse by a factor $4 /\left[1-\left(v_{e} / c\right)^{2}\right]=4 \gamma_{e}^{2}[6]$, where $c$ is the speed of light, $v_{e}$ is the velocity of the ejected electrons, and $\gamma_{e}$ is their relativistic Lorentz factor. In principle, photon energies $\hbar \omega_{0}$ in the $\mathrm{MeV}$ range and beyond can be reached, accompanied by corresponding energy spreads $\sigma$ in the $10-\mathrm{keV}$ range. Backscattering of photons on a flying mirror of electrons has produced coherent photons in the far ultraviolet regime [6] but not yet $\mathrm{MeV}$ photons. Attaining such energies apparently requires a further step. The electrons in the relativistic flying mirror must be compressed to a mean density that is close to condensed-matter values $[11,15]$.

Another proposed production mechanism involves Gamma Factories, more precisely, the atomic degrees of freedom of highly charged ion beams accelerated and stored at CERN [2]. Also here, the main idea is to exploit the relativistic speeds reached at the Large Hadron Collider and harness that energy for production of intense high-energy gamma rays. An optical laser is used to resonantly drive electronic transitions in the highly charged ion beam. Due to the Doppler-effect boost of the laser-photon frequency in the ion's rest frame, that is possible even for highly charged relativistic ions with high atomic number $Z$. Spontaneously emitted photons in the subsequent atomic decay experience an additional boost, such that in the process the initial laser frequency gets amplified by a factor of up to $4 \gamma_{i}^{2}$, where $\gamma_{i}$ is the relativistic Lorentz factor of the ions. At the Large Hadron Collider, this mechanism opens the possibility of producing gamma rays with energies from approximately ten to few hundreds of MeV.

A laser pulse with photon energies comparable to typical nuclear excitation energies is expected to lead to a novel class of nuclear reactions. Multiple absorption of photons will lead to high excitation energies at low spin values, i.e., to states far above the yrast line, because at the photon energies here considered, dipole transitions dominate (the product of mean photon wave number $k_{0}=\omega_{0} / c$ and nuclear radius $R_{N}$ obeys $\left.k_{0} R_{N} \ll 1\right)$. That domain of the nuclear spectrum has not been experimentally accessible so far. Interesting open questions [5] relate to the density of states, to the nuclear equilibration process, and to decay properties. It is likely that multiple neutron evaporation from such states leads to the formation of proton-rich nuclei.

At excitation energies in the $10-\mathrm{MeV}$ range, nuclei are known to equilibrate on very short-time scales. That is expected to be true a forteriori at the excitation energies in the $100-\mathrm{MeV}$ range and beyond that can be reached by multiple absorption of photons with energies of several $\mathrm{MeV}$ each. Rapid equilibration calls for a theoretical treatment of the process in terms of rate equations; see Refs. [3-5]. We derive an expression for the rate by combining the nuclear equilibration process with the description of the laser pulse as a wave packet. We thereby determine the requirements on the laser pulse that must be obeyed to efficiently excite medium-weight and heavy nuclei.

In the main part of the paper we calculate the rate for dipole absorption for a pulse with a typical mean energy $\hbar \omega_{0} \approx 5$ $\mathrm{MeV}$ per photon and with a typical energy spread $\sigma$ in the $10-\mathrm{keV}$ range, using a very general form for the density matrix of the pulse and applying the approximation of a stationary pulse. Specification of that form to either a coherent or an incoherent pulse is deferred until Sec. X. There we show that, all other parameters being equal, the rates for a coherent and an incoherent laser pulse are the same.

We use the following estimates for the characteristic time scales of the process. With $\sigma \approx 10 \mathrm{keV}$, the laser pulse has a spatial extension in flight direction given by $\hbar c / \sigma$, and the interaction time with the target nucleus is $\tau=\hbar / \sigma$. The nuclear equilibration time is given by $\hbar / \Gamma^{\downarrow}$ where the spreading width $\Gamma^{\downarrow}$ is of the order of $5 \mathrm{MeV}$. We show that for a sufficiently intense laser pulse, the induced dipole width $\Gamma^{\text {dip }}=\hbar R$ is also in the $\mathrm{MeV}$ range. We consider the regime $\sigma \ll \Gamma^{\text {dip }}<\Gamma^{\downarrow}$. Each photon absorption process increases the nuclear excitation energy by $\hbar \omega_{0}$ and is quickly followed by internal nuclear equilibration. The consecutive multiple absorption of photons that occurs during the interaction time $\tau$ is described by a rate equation [5] with the rate $R=\Gamma^{\mathrm{dip}} / \hbar$ as input. We derive an expression for $R$ for a single dipole absorption process that starts either from the ground state or from an equilibrated excited state of the target nucleus.

To calculate the single photon absorption process, we expand the electromagnetic field (and, in its wake, the density matrix of the laser pulse) in a basis of orthonormal states. These states are defined in a cube of side length $L$ and by periodic boundary conditions. The target nucleus is located at the center $\vec{r}=0$ of the cube. The laser pulse carrying $N$ photons is described by means of the density matrix as a wave 
packet that traverses the cube. Dipole excitation takes place during the time $\tau$ when the wave packet overlaps the target nucleus. For that picture to apply, the side length $L$ of the cube must obviously be large compared to the linear dimensions of the wave packet. We eventually take the limit $L \rightarrow \infty$.

\section{TRANSITION PROBABILITY AND RATE}

Let $\vec{j}$ be the operator of the nuclear current density and $\vec{A}$ be the vector potential of the electromagnetic field. In Coulomb gauge $\left(A_{4}=0, \operatorname{div} \vec{A}=0\right)$, the interaction Hamiltonian is

$$
H=-\frac{1}{c} \vec{j} \cdot \vec{A}
$$

We use the interaction representation. Then $\vec{j}$ is time dependent and carries the factor $\exp \left\{i \omega_{f i} t\right\}$, with $\omega_{f i}=\left(E_{f}-\right.$ $\left.E_{i}\right) / \hbar>0$ and $E_{f}\left(E_{i}\right)$ the energies of the initial and final nuclear states, respectively. We expand $\vec{A}$ in a set of orthonormal modes, defined in a large but finite cubic quantization volume of side length $L$ with periodic boundary conditions and the target nucleus at its center. The modes are polarized plane waves $L^{-3 / 2} \vec{e}_{\lambda} \exp \left\{i \vec{k} \vec{r}-i \omega_{k} t\right\}$ with discrete wave vectors $\vec{k}=$ $\left\{k_{x}, k_{y}, k_{z}\right\}$ and with $\omega_{k}=c|\vec{k}|$. The two (real) polarization vectors $\vec{e}_{\lambda}(\vec{k})$ with $\lambda= \pm 1$ are orthogonal upon $\vec{k}$ and upon each other. Then

$$
\vec{A}=\sum_{\lambda, \vec{k}} \frac{c}{i \omega_{k}} \sqrt{\frac{2 \pi \hbar \omega_{k}}{L^{3}}} \vec{e}_{\lambda}(\vec{k})\left[a_{\vec{k} \lambda} \exp \left\{i \vec{k} \vec{r}-i \omega_{k} t\right\}-\text { H.c. }\right]
$$

Upon quantization, the expansion coefficients $a_{\vec{k}, \lambda}^{\dagger}$ and $a_{\vec{k}, \lambda}$ become bosonic creation and annihilation operators, respectively. For simplicity we label each mode by $k=(\vec{k}, \lambda)$.

The rate for a dipole transition induced by $H(t)$ is calculated in second-order perturbation theory. We start with the time-dependent transition amplitude. To first order as a function of time $T$ it is given by $[1 /(i \hbar)] \int_{0}^{T} \mathcal{M}_{\left(i_{P}, i_{N}\right) \rightarrow\left(f_{P}, f_{N}\right)}(t) d t$, where the matrix element

$$
\mathcal{M}_{\left(i_{P} i_{N}\right) \rightarrow\left(f_{P} f_{N}\right)}(t)=\left\langle f_{P} ; f_{N} J_{f} M_{f}|H(t)| i_{N} J_{i} M_{i} ; i_{P}\right\rangle
$$

is the amplitude for the transition from an initial state $\left(i_{N}, i_{P}\right)$ to a final state $\left(f_{N}, f_{P}\right)$. Explicitly, the initial state $\left|i_{N} J_{i} M_{i} ; i_{P}\right\rangle$ is the product of the initial nuclear state $\left|i_{N}\right\rangle$ with energy $E_{i}$, spin $J_{i}$, and magnetic quantum number $M_{i}$, and of the initial state $\left|i_{P}\right\rangle$ of the photon field, and correspondingly for the final state $\left|f_{N} J_{f} M_{f} ; f_{P}\right\rangle$. The final nuclear state $\left|f_{N}\right\rangle$ has energy $E_{f}$, spin $J_{f}$, and magnetic quantum number $M_{f}$. The final photon state $\left|f_{P}\right\rangle$ corresponds to the field after the absorption of one photon. The transition amplitude (3) factorizes, one factor describing the transition of the photon field, the other, the nuclear transition.

For our semiquantitative estimate of the rate, we parametrize (the square of) the nuclear transition matrix element using the Brink-Axel hypothesis. That is done in Sec. VIII. There we also address the limitations of our approach. Use of the Brink-Axel hypothesis makes it unnecessary to introduce a specific model for initial and final nuclear states. Such models can be found, for instance, in Ref. [16]. For the photon field we use Fock states with fixed photon number. ${ }^{1}$ For mode $k$, the normalized state carrying $n_{k}$ photons, with $n_{k}=0,1, \ldots$, is

$$
\left|n_{k}\right\rangle=\left(n_{k} !\right)^{-1 / 2}\left(a_{k}^{\dagger}\right)^{n_{k}}|0\rangle
$$

with $|0\rangle$ the photon vacuum. In the Hilbert space of orthonormal multiphoton states $|i\rangle=\left|n_{1}^{(i)}\right\rangle\left|n_{2}^{(i)}\right\rangle \times \cdots \times\left|n_{M}^{(i)}\right\rangle$, with $i=1,2, \ldots$ and $\langle i \mid j\rangle=\delta_{i j}$, each state $|i\rangle$ is the product of $M$ modes, each mode carrying $n_{k}^{(i)}$ photons. We consider an incident laser pulse carrying $N$ photons and use the notation $\left|i_{P}\right\rangle=|i, N\rangle$ for the initial photonic state. The final photon state after absorption of a photon is then $\left|f_{P}\right\rangle=|f, N-1\rangle$.

The total transition probability $P(T)$ is obtained in second order perturbation theory by averaging (summing) the product $\int_{0}^{T} d t \mathcal{M}_{\left(i_{P}, i_{N}\right) \rightarrow\left(f_{P}, f_{N}\right)}(t) \int_{0}^{T} d t^{\prime} \mathcal{M}_{\left(i_{P}, i_{N}\right) \rightarrow\left(f_{P}, f_{N}\right)}^{*}\left(t^{\prime}\right)$ over the initial (final) photonic and nuclear states. Following common usage [17] we describe the incoming laser pulse in terms of a density matrix $\rho_{N}$. That matrix is built from photon states $|i, N\rangle$ each carrying $N$ photons so that $\sum_{k}\left\langle i, N\left|a_{k}^{\dagger} a_{k}\right| i, N\right\rangle=$ $\sum_{k} n_{k}^{(i)}=N$ for all $i=1, \ldots$. Within that framework the most general expression for the density matrix is

$$
\rho_{N}=\sum_{i j} \rho_{i j}|i, N\rangle\langle j, N| \text {. }
$$

Here $\rho_{i j}^{*}=\rho_{j i}$, and $\sum_{i} \rho_{i i}=1$. The coefficients $\rho_{i j}$ must be chosen such as to best model the shape of the incident laser pulse (i.e., of the "wave packet" mentioned in Sec. II).

With the initial distribution of photon states given by the density matrix (5), the total transition probability $P(T)$ reads

$$
\begin{aligned}
P(T)= & \frac{1}{(\hbar)^{2}} \int_{0}^{T} d t \int_{0}^{T} d t^{\prime} \frac{1}{2 J_{i}+1} \sum_{f_{N} f_{P}} \sum_{M_{i} J_{f} M_{f}} \sum_{i j} \rho_{i j} \\
& \times \mathcal{M}_{\left(i_{P} i_{N}\right) \rightarrow\left(f_{P} f_{N}\right)}(t) \mathcal{M}_{\left(j_{P} i_{N}\right) \rightarrow\left(f_{P} f_{N}\right)}^{*}\left(t^{\prime}\right) .
\end{aligned}
$$

The sum over $\left(f_{N} J_{f}\right)$ extends over all nuclear states that can be reached via dipole absorption from the single initial nuclear state $\left(i_{N} J_{i}\right)$. The sum over $f_{P}$ comprises the states $|f, N-1\rangle$. As shown below, for sufficiently large times $T, P(T)$ is linear in $T$. That fact allows for a definition of the rate $R$ and of the dipole width $\Gamma_{\text {dip }}$ given by

$$
R=\frac{P(T)}{T}, \quad \Gamma_{\mathrm{dip}}=\hbar \frac{P(T)}{T} .
$$

In the following sections we evaluate and discuss the expressions for $P(T), R$, and $\Gamma_{\text {dip }}$. These are the objects of central interest. We now outline these steps.

We start by evaluating the photonic part of the matrix element in Sec. IV. We use a stationarity condition for the density matrix that is justified later in Sec. IX. In Sec. V

\footnotetext{
${ }^{1}$ Our approach uses photon states $|i, N\rangle$ with fixed photon number $N$ throughout, while Glauber [17] considers coherent states that do not have fixed photon number. Common to both approaches is the assumption of stationarity. Both approaches lead to identical expressions for the rate as given in Eq. (30), with $N$ in our case replaced for the case of coherent states by the mean photon number $\langle N\rangle$ in the pulse. Our definition of coherence in Sec. X is consistent with Glauber's.
} 
we use the result so obtained in the expression for $P(T)$. For the evaluation of the nuclear part of the matrix element we simplify the operator $\vec{j}$ of the current density using the dipole approximation in the long wavelength limit. We use the Wigner-Eckart theorem to introduce the reduced nuclear dipole matrix element. We perform the two time integrations and sum over $\left(M_{i}, M_{f}\right)$. For the remaining sum over $f_{N}$ we use in Sec. VI the Brink-Axel hypothesis. In Sec. VII we correct an oversimplification introduced via the stationarity condition in Sec. IV, and we perform the ensuing additional summation over photon states that was omitted in Sec. IV. The resulting final expression for the rate is discussed in Sec. VIII.

In the main part of the paper we use the general expression (5) for the density matrix of the laser pulse. That expression allows for the pulse to be fully or partially coherent, or to lack coherence altogether. Therefore, the resulting expression for the absorption rate applies irrespective of the degree of coherence of the laser pulse. That fact is confirmed, and the reason is analyzed, in Sec. X. In Sec. XI we show that our result for the rate applies also for short times and can, therefore, be used in rate equations.

\section{TRANSITION PROBABILITY IN PHOTON SPACE}

For clarity of presentation we first deal with the transition induced by the operator (1) in photon space only. Anticipating that the transition time $\hbar / \Gamma_{\text {dip }}$ calculated within our approach is very short in comparison with the duration time $\hbar / \sigma$ of the laser pulse, we assume that the laser pulse is stationary. Obviously, that assumption strictly applies only for a laser beam which is infinitely extended in time. It holds only approximately for the actual duration time $\hbar / \sigma$ of the laser pulse. In Sec. IX we show that, except for factors of order unity, our result for the rate holds even for the short times characteristic of multiple nuclear photon excitation due to a single laser pulse.

We write the vector potential in Eq. (2) as the sum of two components, $\vec{A}^{+}\left(\vec{A}^{-}\right)$carrying the annihilation operators (the creation operators, respectively),

$$
\vec{A}=\sum_{k}\left(\vec{A}_{k}^{+} a_{k}+\vec{A}_{k}^{-} a_{k}^{\dagger}\right) .
$$

Inserting Eq. (8) into expressions (3) and (6) and using the notation introduced in the equation above, we find that the transition probability from an initial state $|i, N\rangle$ to a final state $|f, N-1\rangle$ is given by

$$
\sum_{k l} \vec{A}_{l}^{+} \vec{A}_{k}^{-}\left\langle f, N-1\left|a_{l} \rho_{N} a_{k}^{\dagger}\right| f, N-1\right\rangle .
$$

To evaluate this term we use the general definition (5) and write the density matrix as the sum of three terms,

$$
\rho_{N}=\rho_{N}^{\text {diag }}+\rho_{N}^{(1)}+\rho_{N}^{(2)} .
$$

Here $\rho_{N}^{\text {diag }}=\sum_{i} \rho_{i i}|i, N\rangle\langle i, N|$ is the diagonal contribution. The term $\rho_{N}^{(1)}$ contains pairs of states $i \neq j$ such that state $|j, N\rangle$ is obtained from state $|i, N\rangle$ by transferring a single photon from some mode to another mode. The term $\rho_{N}^{(2)}$ contains pairs of states $i \neq j$ such that state $|j, N\rangle$ is obtained from state $|i, N\rangle$ by the transfer of at least two photons. Inspection shows that the term $\rho_{N}^{(2)}$ in Eq. (10) does not contribute to Eq. (9): No final state $|f, N-1\rangle$ exists that could be reached via absorption of a single photon from both states $|i, N\rangle$ and $|j, N\rangle$ occurring pairwise in $\rho_{N}^{(2)}$. That statement does not apply to $\rho_{N}^{(1)}$. However, the physical parameters of the problem allow for a further simplification based on stationarity.

The time during which the pulse interacts with the target nucleus located at $\vec{r}=0$ (the "length in time" of the pulse) is $\tau=\hbar / \sigma$. We assume that $\tau$ is large compared with the characteristic time $\hbar / \Gamma_{\text {dip }}$ of dipole excitation. It is then reasonable to take the density matrix for the laser pulse as (almost) stationary. That implies [17]

$$
\operatorname{Tr}\left[\rho_{N} a_{k}^{\dagger} a_{l}\right]=\delta_{k l} \bar{n}_{k} .
$$

The coefficient $n_{k}$ gives the mean photon number in the mode $k$ of the density matrix $\rho_{N}$. Intuitively speaking, condition (11) rules out contributions that would give rise to an oscillatory time dependence of the form $\exp \left\{i\left(\omega_{k}-\omega_{l}\right) t\right\}$, violating the stationarity condition. Rather than a constraint on the density matrix, Eq. (11) actually defines the time scale beyond which conclusions based on stationarity apply; see Sec. IX.

With the stationarity condition (11) the only part of the density matrix $\rho_{N}$ that gives a nonzero contribution to the transition probability is $\rho_{N}^{\mathrm{diag}}=\sum_{i} \rho_{i i}|i, N\rangle\langle i, N|$. For a single term in that sum, we calculate the transition probability to any final state $|f, N-1\rangle$ carrying $N-1$ photons. For $\vec{A}^{+}\left(\vec{A}^{-}\right)$ we first consider in the sum (8) the contribution due to a single term carrying the label $k$ (the label $l$, respectively). The transition probability is given by $\left\langle f, N-1\left|a_{k}\right| i, N\right\rangle\left\langle i, N\left|a_{l}^{\dagger}\right| f, N-\right.$ $1\rangle$. For $k \neq l$, the probability vanishes for every state $\mid f, N-$ $1\rangle$. For $k=l$ it vanishes for $n_{k}^{(i)}=0$. For $n_{k}^{(i)} \geqslant 1$ there is exactly one state $|f(i, k), N-1\rangle=\left(n_{k}^{(i)}\right)^{-1 / 2} a_{k}|i, N\rangle$ for which the probability does not vanish. The resulting transition probability is $\delta_{k l} n_{k}^{(i)}$ for all $n_{k}^{(i)} \geqslant 0$. The total transition probability due to photon absorption from the state $|i, N\rangle$ is

$$
\begin{aligned}
& \sum_{k l}\left\{\vec{A}_{k}^{+} \vec{A}_{l}\left\langle f(i, k), N-1\left|a_{k}\right| i, N\right\rangle\left\langle i, N\left|a_{l}^{\dagger}\right| f(i, k), N-1\right\rangle\right\} \\
& \quad=\sum_{k} n_{k}^{(i)} \vec{A}_{k}^{+} \vec{A}_{k} .
\end{aligned}
$$

Each transition in the sum (12) involves a single final state $|f(i, k), N-1\rangle$ only. The photon part of the transition probability for a general stationary density matrix (5) obeying Eq. (11) is

$$
\sum_{k} \sum_{i} \rho_{i i} n_{k}^{(i)} \vec{A}_{k}^{+} \vec{A}_{k}=\sum_{k} \vec{n}_{k} \vec{A}_{k}^{+} \vec{A}_{k} .
$$

Equation (13) provides the most general expression of the transition probability in photon space for a stationary laser pulse. The coefficients $\bar{n}_{k}=\sum_{i} \rho_{i i} n_{k}^{(i)}$ are the average occupation probabilities of the mode $k$ in the laser pulse. They obey

$$
\sum_{k} \bar{n}_{k}=N
$$


With increasing side length $L$ of the normalization volume in Eq. (2), the density of modes increases. The number of terms in the sums (13) and (14) increases likewise while the average occupation numbers $\bar{n}_{k}$ decrease. The invariant and physically meaningful quantity here is $N$, the total number of photons in the pulse.

In quantum optics the photoabsorption process often involves optical photons in a finite cavity. Then the number of photons $N$ is replaced by the photon density in the cavity $[18,19]$. However, that approach is less appropriate in our case which lacks a cavity volume. In the discussion in Sec. VIII we identify the parameter equivalent to the photon density and, thereby, display agreement with the quantum-optics approach.

\section{TOTAL TRANSITION PROBABILITY}

We return to the total transition probability $P(T)$ in Eq. (6), using the result (13) and the full notation $k \rightarrow(\vec{k}, \lambda)$. To keep the notation simple we first suppress spin and magnetic quantum numbers in the nuclear wave functions. The matrix elements in the integrand of Eq. (6) are written as

$$
\begin{aligned}
& \sum_{\vec{k}, \lambda} \frac{2 \pi \hbar}{\omega_{k} L^{3}} \bar{n}_{\vec{k}, \lambda}\left\langle i_{N}\left|\vec{j}^{\prime \prime} \vec{e}_{\lambda}(\vec{k}) \exp \left\{-i \vec{k} \vec{r}^{\prime}+i \omega_{k} t^{\prime}\right\}\right| f_{N}\right\rangle \\
& \quad \times\left\langle f_{N}\left|\vec{j} \vec{e}_{\lambda}(\vec{k}) \exp \left\{i \vec{k} \vec{r}-i \omega_{k} t\right\}\right| i_{N}\right\rangle .
\end{aligned}
$$

The operator $\vec{j}\left(\vec{j}^{\prime}\right)$ of the current density depends only on the unprimed variables (the primed variables, respectively). We confine ourselves to electric dipole transitions. We use the Siegert theorem [20] and the long wavelength limit for each of the two factors in big round brackets. We recall that the target nucleus is at the centers $\vec{r}=0=\vec{r}^{\prime}$ of the two coordinate systems. In cgs (centimeter-gram-seconds) units, expression (15) then becomes

$$
\begin{aligned}
& \sum_{\vec{k}, \lambda} \frac{8 \pi^{2}}{3} \frac{e^{2} \hbar \omega_{k}}{L^{3}} \bar{n}_{\vec{k}, \lambda}\left\langle i_{N}\left|r^{\prime} Y_{1}^{\lambda *}\left(\vec{k} \mid \Omega^{\prime}\right)\right| f_{N}\right\rangle \\
& \quad \times\left\langle f_{N}\left|r Y_{1}^{\lambda}(\vec{k} \mid \Omega)\right| i_{N}\right\rangle \exp \left\{i\left(\omega_{k}-\omega_{f i}\right)\left(t^{\prime}-t\right)\right\} .
\end{aligned}
$$

We have simplified the notation by suppressing sums over proton and neutron coordinates carrying effective charges. That fact is properly taken into account in the order-ofmagnitude estimate given below in Sec. VIII. For each term in the sum over $(\vec{k}, \lambda)$ the spherical harmonic $Y_{1}^{\lambda}(\vec{k} \mid \Omega)$ is defined with respect to a Cartesian coordinate system spanned by the vectors $\vec{k}, \overrightarrow{e_{1}}, \overrightarrow{e_{2}}$, with $\vec{k}$ pointing in the direction of the $z$ axis. The magnetic quantum numbers $\lambda= \pm 1$ correspond to the polarization vectors $\vec{e}_{\lambda}(\vec{k})$ in Eq. (2). The argument $\Omega$ comprises polar and azimuthal angles in that system. The occupation numbers $\bar{n}_{\vec{k}, \lambda}$ restrict the summation over $\vec{k}$ in Eq. (16) by the aperture $\alpha$ of the laser pulse. Throughout the paper we assume that the pulse is well collimated so that $\alpha \ll 1$. Then, the directions of the vectors $\vec{k}$ differ by less than $\alpha$ from each other and from the mean direction $\vec{k}_{0}$ of the laser pulse, defined as

$$
\vec{k}_{0}=\sum_{\vec{k}, \lambda} \bar{n}_{\vec{k}, \lambda} \vec{k}
$$

Without loss of generality we assume that, for each value of $\lambda= \pm 1$, the directions of the unit vectors $\vec{e}_{\lambda}(\vec{k})$ also differ by less than $\alpha$ from each other and from suitably defined vectors $\vec{e}_{\lambda}\left(\vec{k}_{0}\right)$ that are orthogonal upon $\vec{k}_{0}$ and their scalar product obeys $\left(\vec{e}_{1}\left(\vec{k}_{0}\right), \vec{e}_{2}\left(\vec{k}_{0}\right)\right)=0$. The nuclear states with initial (final) spins $J_{i}\left(J_{f}\right)$ and $z$ components $M_{i}\left(M_{f}\right)$ are quantized in the coordinate system spanned by $\vec{k}_{0}, \vec{e}_{1}\left(\vec{k}_{0}\right), \vec{e}_{2}\left(\vec{k}_{0}\right)$. The nuclear matrix element $\left\langle f_{N}\left|r Y_{1}^{\lambda}(\vec{k} \mid \Omega)\right| i_{N}\right\rangle$ is evaluated by rotating the spherical harmonic $Y_{1}^{\lambda}$ so that the quantization axis coincides with the direction $\vec{k}_{0}$ of nuclear quantization. Using Wigner $D$ functions [21] we have

$$
Y_{1}^{\lambda}(\vec{k} \mid \Omega)=\sum_{\mu} Y_{1}^{\mu}\left(\vec{k}_{0} \mid \zeta\right) D_{\mu \lambda}^{1}
$$

The arguments $\Omega$ and $\zeta$ are connected by the rotation. The arguments of $D_{\mu \lambda}^{1}$ are the Euler angles characterizing the rotation that carries the Cartesian coordinate system spanned by the vectors $\vec{k}, \vec{e}_{1}(\vec{k}), \vec{e}_{2}(\vec{k})$ to the system spanned by the vectors $\vec{k}_{0}, \vec{e}_{1}\left(\vec{k}_{0}\right), \vec{e}_{2}(\vec{k})_{0}$. Every one of these angles is bounded by $\alpha$. The angular dependence of $D_{\mu \lambda}^{1}$ is simple and involves only $\sin$ and cos functions. These change over a typical range of $\pi / 2$. Since $\alpha \ll \pi / 2$ we may, to leading order in $\alpha$, then replace $Y_{1}^{\lambda}(\vec{k} \mid \Omega) \rightarrow Y_{1}^{\lambda}\left(\vec{k}_{0} \mid \Omega\right)$.

We return to the full notation of the nuclear states. Expression (16) becomes

$$
\begin{aligned}
& \sum_{\vec{k}, \lambda} \frac{8 \pi^{2}}{3} \frac{e^{2} \hbar \omega_{k}}{L^{3}} \bar{n}_{\vec{k}, \lambda}\left|\left\langle f_{N} J_{f} M_{f}\left|r Y_{1}^{\lambda}\left(\vec{k}_{0} \mid \Omega\right)\right| i_{N} J_{i} M_{i}\right\rangle\right|^{2} \\
& \quad \times \exp \left\{i\left(\omega_{k}-\omega_{f i}\right)\left(t^{\prime}-t\right)\right\} .
\end{aligned}
$$

We apply the Wigner-Eckart theorem [21] introducing the reduced nuclear matrix element (indicated by a double bar) and perform the average (sum) over initial (final) nuclear magnetic quantum numbers. The probability $P_{f_{N} i_{N}}(T)$ at time $T$ for the particular dipole transition $\left|i_{N}\right\rangle \rightarrow\left|f_{N}\right\rangle$ is obtained by integrating expression (19) over $t$ and $t^{\prime}$ from zero to $T$ and multiplying by $\hbar^{-2}$,

$$
\begin{aligned}
P_{f_{N} i_{N}}(T)= & \frac{8 \pi^{2}}{3^{2}} \frac{e^{2}}{\hbar c} \sum_{\vec{k}, \lambda} \frac{\omega_{k} c}{L^{3}} \bar{n}_{\vec{k}, \lambda}\left[4 \frac{\sin ^{2}\left[\left(\omega_{k}-\omega_{f i}\right) T / 2\right]}{\left(\omega_{k}-\omega_{f i}\right)^{2}}\right] \\
& \times\left|\left\langle i_{N} J_{i}|| r Y_{1}\left(\vec{k}_{0} \mid \Omega\right)|| f_{N} J_{f}\right\rangle\right|^{2} .
\end{aligned}
$$

Expression (20) for $P_{f_{N} i_{N}}(T)$ holds for a stationary laser pulse with sufficiently small aperture and for a dipole transition in the long wavelength limit. Because of the sum over $\vec{k}$ and $\lambda$, Eq. (20) gives the average transition probability for dipole absorption.

The total transition probability is obtained by summing expression (20) over the final nuclear states $\left(f_{N} J_{f}\right)$. That sum is carried out using the Brink-Axel hypothesis in Sec. VI. Expression (20) vanishes in the continuum limit $L \rightarrow \infty$ where $\bar{n}_{\vec{k}, \lambda} \rightarrow 0$. That is a consequence of considering a stationary pulse via Eq. (11). We show in Sec. IX that the rate is actually obtained by summing expression (20) over the photon states occupied in the primary laser pulse. We refer to that sum as to a sum over final states (which is actually a misnomer). We thereby follow common usage. The expression is used, for 
instance, in the standard derivation of Fermi's "golden rule" as discussed in many textbooks on quantum mechanics; see, for instance, Ref. [22]. This sum over photon states is carried out in Sec. VII.

\section{SUM OVER FINAL NUCLEAR STATES}

The summation over final nuclear states $\left(f_{N} J_{f}\right)$ in Eq. (6) is carried out using the Brink-Axel hypothesis [23,24]. The hypothesis states that dipole absorption from any initial nuclear state $i_{N}$ (ground or excited state) populates preferentially the GDR built upon that state. The GDR is the normalized mode $d\left(i_{N}\right)$ obtained by applying the dipole operator to the initial state $i_{N}$. The GDR is not an eigenstate of the nuclear Hamiltonian nor of total spin or isospin. It is a mode that is shared by a large number of eigenstates. The probability distribution of the GDR over the eigenstates $\left(f_{N} J_{f}\right)$ of the nuclear Hamiltonian at energies $E_{f}$ is described by a normalized Lorentzian $\Gamma^{\downarrow} /\left\{(2 \pi)\left[\left(E_{f}-E_{d}\right)^{2}+(1 / 4)\left(\Gamma^{\downarrow}\right)^{2}\right]\right\}$. We consider the same GDR expression for all final spin values $J_{f}$ and, if applicable, for all final isospin values. The mean energy $E_{d}$ of the GDR is defined as the expectation value of the nuclear Hamiltonian for the mode $d\left(i_{N}\right)$, with typical values $E_{d}-E_{i} \approx 12 \mathrm{MeV}$ for medium-weight and $E_{d}-E_{i} \approx 8 \mathrm{MeV}$ for heavy nuclei. Nuclear dissipation is characterized by the spreading width $\Gamma^{\downarrow} \approx 5 \mathrm{MeV}$ introduced in Sec. II. We accordingly use the replacement

$$
\begin{aligned}
& \left|\left\langle i_{N} J_{i}|| r Y_{1}\left(\vec{k}_{0} \mid \Omega\right)|| f_{N} J_{f}\right\rangle\right|^{2} \rightarrow\left|\left\langle i_{N} J_{i}|| r Y_{1}\left(\vec{k}_{0} \mid \Omega\right)|| d\left(i_{N}\right) J_{f}\right\rangle\right|^{2} \\
& \quad \times \frac{\Gamma^{\downarrow}}{2 \pi\left[\left(E_{f}-E_{d}\right)^{2}+(1 / 4)\left(\Gamma^{\downarrow}\right)^{2}\right]} .
\end{aligned}
$$

We insert that into Eq. (20) and integrate over final energies $E_{f}$. That gives

$$
\begin{aligned}
P_{i_{N} \rightarrow d\left(i_{N}\right)}(T) \\
=\frac{8 \pi^{2}}{3^{2}} \frac{e^{2}}{\hbar c} \sum_{\vec{k}, \lambda} \frac{\omega_{k} c}{L^{3}} \bar{n}_{\vec{k}, \lambda} \sum_{J_{f}}\left|\left\langle i_{N} J_{i}|| r Y_{1}\left(\vec{k}_{0} \mid \Omega\right)|| d\left(i_{N}\right) J_{f}\right\rangle\right|^{2} \\
\quad \times \int_{E_{d}-2 \Gamma^{\downarrow}}^{E_{d}+2 \Gamma^{\downarrow}} d E_{f} \frac{\Gamma^{\downarrow}}{2 \pi\left[\left(E_{f}-E_{d}\right)^{2}+(1 / 4)\left(\Gamma^{\downarrow}\right)^{2}\right]} \\
\quad \times 4 \frac{\sin ^{2}\left[\left(\omega_{k}-\omega_{f i}\right) T / 2\right]}{\left(\omega_{k}-\omega_{f i}\right)^{2}} .
\end{aligned}
$$

In the interval $\left[E_{d}-2 \Gamma^{\downarrow}, E_{d}+2 \Gamma^{\downarrow}\right]$, the Lorentzian provides a semiquantitative description of the spreading of the GDR over the eigenstates of the nuclear Hamiltonian. The distant tails of the Lorentzian are not physically relevant. For better control over the approximations that are to follow, we have indicated that fact by assigning these limits to the integration over $E_{f}$. The sum over $J_{f}$ extends over the spin values that can be reached via dipole absorption from the initial spin value $J_{i}$. A possible sum over final isospin values is suppressed.

Clearly the assumption of a Lorentzian in Eq. (21) which is, moreover, common to all final spin and isospin values is a rough approximation to reality. If the initial state is the ground state, that approximation can be much improved with the help of more detailed nuclear models and/or refined theoretical approaches. The literature on the subject is very extensive. Without any claim to completeness we mention Chaps. 8.3.3 and 8.5 in the book by Ring and Schuck [16] where the GDR is treated in the framework of the nuclear shell model and, by way of example, Refs. [25-31]. These discuss the influence on GDR properties of Landau damping, of nuclear ground-state deformation, of the Skyrme force, of the pairing force within various versions of the random-phase approximation, and/or of the nuclear density functional. It would be premature to address such issues here, for two reasons. First, it is not clear (and probably not known) if and how such nuclear-structure properties would affect the GDR built upon states $\left|i_{N} J_{i}\right\rangle$ far above yrast. Second, as discussed in the introductory section, our approach is based on the Brink-Axel hypothesis, whose validity far above yrast has not been established so far. This is why we aim at a semiquantitative estimate for the rate.

We define the mean frequency $\omega_{0}$ of the laser pulse by

$$
\omega_{0}=\frac{\sum_{\vec{k}, \lambda} \bar{n}_{\vec{k}, \lambda} \omega_{k}}{\sum_{\vec{k}, \lambda} \bar{n}_{\vec{k}, \lambda}} .
$$

The occupation numbers $\bar{n}_{\vec{k}, \lambda}$ and the frequencies $\omega_{k}$ are concentrated within a frequency interval of width $\sigma / \hbar$ centered at $\omega_{0}$. That interval is very small compared to $\Gamma^{\downarrow} / \hbar$. Likewise, the square of the Bessel function in Eq. (22) is, for values of $T \gg \hbar / \Gamma^{\downarrow}$, sharply peaked at $\omega_{k}=\omega_{f i}$. In contradistinction and because of the large value of the spreading width, the Lorentzian factor under the integral is a very smooth function of $E_{f}$. Therefore, it is legitimate to pull the Lorentzian out from under the integral, replacing $E_{f} \rightarrow E_{i}+\hbar \omega_{0}$. Thus,

$$
\begin{aligned}
P_{i_{N} \rightarrow d\left(i_{N}\right)}(T)= & \frac{8 \pi^{2}}{3^{2}} \frac{e^{2}}{\hbar c} \sum_{J_{f}}\left|\left\langle i_{N} J_{i}|| r Y_{1}\left(\vec{k}_{0} \mid \Omega\right)|| d\left(i_{N}\right) J_{f}\right\rangle\right|^{2} \\
& \times \frac{\Gamma^{\downarrow}}{2 \pi\left[\left(E_{i}+\hbar \omega_{0}-E_{d}\right)^{2}+(1 / 4)\left(\Gamma^{\downarrow}\right)^{2}\right]} \\
& \times \sum_{\vec{k}, \lambda} \frac{\omega_{k} c}{L^{3}} \bar{n}_{\vec{k}, \lambda} \\
& \times \int_{E_{d}-2 \Gamma^{\downarrow}}^{E_{d}+2 \Gamma^{\downarrow}} d E_{f} 4 \frac{\sin ^{2}\left[\left(\omega_{k}-\omega_{f i}\right) T / 2\right]}{\left(\omega_{k}-\omega_{f i}\right)^{2}} .
\end{aligned}
$$

We assume that $\omega_{0}$ is located well within the integration interval. The same is then true for each one of the $\omega_{k}$ values. It is, thus, legitimate for $T \gg \hbar / \Gamma^{\downarrow}$ to extend the integral over $E_{f}$ from $-\infty$ to $+\infty$. Then every one of the resulting integrals in the sum over $\vec{k}, \lambda$ (each one carrying a different variable $\omega_{k}$ ) has the value $2 \pi \hbar T$. With the help of Eqs. (14) and (23), the sum over $(\vec{k}, \lambda)$ can be carried out. We obtain

$$
\begin{aligned}
P_{i_{N} \rightarrow d\left(i_{N}\right)}(T)= & \frac{8 \pi^{2}}{3^{2}} \frac{e^{2}}{\hbar c} \sum_{J_{f}}\left|\left\langle i_{N} J_{i}|| r Y_{1}\left(\vec{k}_{0} \mid \Omega\right)|| d\left(i_{N}\right) J_{f}\right\rangle\right|^{2} \\
& \times \frac{\Gamma^{\downarrow}}{\left(E_{i}+\hbar \omega_{0}-E_{d}\right)^{2}+(1 / 4)\left(\Gamma^{\downarrow}\right)^{2}} \\
& \times c T N \hbar \omega_{0} \frac{1}{L^{3}} .
\end{aligned}
$$


The transition rate $R$ (transition probability per unit time) becomes

$$
\begin{aligned}
R_{i_{N} \rightarrow d\left(i_{N}\right)}= & \frac{8 \pi^{2}}{3^{2}} \frac{e^{2}}{\hbar c} \frac{N \hbar \omega_{0} c}{L^{3}} \sum_{J_{f}}\left|\left\langle i_{N} J_{i}|| r Y_{1}\left(\vec{k}_{0} \mid \Omega\right) \| d\left(i_{N}\right) J_{f}\right\rangle\right|^{2} \\
& \times \frac{\Gamma^{\downarrow}}{\left(E_{i}+\hbar \omega_{0}-E_{d}\right)^{2}+(1 / 4)\left(\Gamma^{\downarrow}\right)^{2}} .
\end{aligned}
$$

\section{SUM OVER FINAL PHOTON STATES}

The rate (26) depends via the factor $L^{-3}$ upon the (unphysical) quantization volume. Moreover, the rate vanishes in the limit $L \rightarrow \infty$. That is because we have used the stationarity condition (11). As shown in Sec. IX, the Kronecker delta in that condition is physically meaningful only if the resulting expression for the rate is summed over the photon states $(\vec{k}, \lambda)$ occupied in the laser pulse. For $L \rightarrow \infty$, the number of such states grows like $L^{3}$, compensating the factor $L^{-3}$ in Eq. (26). That gives

$$
\begin{aligned}
R= & \sum_{\vec{k}, \lambda} R_{i_{N} \rightarrow d\left(i_{N}\right)} \\
= & \frac{8 \pi^{2}}{3^{2}} \frac{e^{2}}{\hbar c} N \hbar \omega_{0} c \sum_{J_{f}}\left|\left\langle i_{N} J_{i}|| r Y_{1}\left(\vec{k}_{0}|| \Omega\right) \mid d\left(i_{N}\right) J_{f}\right\rangle\right|^{2} \\
& \times \frac{\Gamma^{\downarrow}}{\left(E_{i}+\hbar \omega_{0}-E_{d}\right)^{2}+\left(\Gamma^{\downarrow}\right)^{2}} \sum_{\vec{k}, \lambda} \frac{1}{L^{3}} .
\end{aligned}
$$

We use the identity (valid for $L \rightarrow \infty$ ) [18,19]

$$
\sum_{\vec{k}, \lambda} \frac{1}{L^{3}} \rightarrow \frac{2}{(2 \pi)^{3}} \int d^{3} k
$$

The factor 2 accounts for the two directions of polarization. As mentioned at the end of Sec. V and as explained in detail in Sec. IX, the integral runs over the photon states occupied in the incident laser pulse. These states comprise a segment of a shell in three-dimensional $k$ space with central radius $k_{0}=\left|\vec{k}_{0}\right|=\left|\omega_{i f}\right| / c$, thickness $\delta k=\sigma /(\hbar c)$, and aperture $\alpha \ll \pi / 2$. These parameters obviously depend on the way the pulse is generated. We use spherical polar coordinates. The integral over solid angle yields $2 \pi \alpha$, and for $\delta k \ll k_{0}$ the right-hand side of expression (28) becomes

$$
\frac{2 \alpha}{(2 \pi)^{2}} \int_{k_{0}-\delta k / 2}^{k_{0}+\delta k / 2} d k k^{2} \approx \frac{\alpha}{2 \pi^{2}} k_{0}^{2} \delta k .
$$

Thus,

$$
\begin{aligned}
R= & \frac{1}{9 \pi} \frac{e^{2}}{\hbar} \sum_{J_{f}}\left|\left\langle i_{N} J_{i}|| r Y_{1}\left(\vec{k}_{0}|| \Omega\right) \mid d\left(i_{N}\right) J_{f}\right\rangle\right|^{2} \\
& \times \frac{\Gamma^{\downarrow}}{\left(E_{i}+\hbar \omega_{0}-E_{d}\right)^{2}+(1 / 4)\left(\Gamma^{\downarrow}\right)^{2}}\left[N \hbar \omega_{0} 4 \pi \alpha k_{0}^{2} \delta k\right] .
\end{aligned}
$$

Expression (30) for the transition rate $R$ holds for a stationary laser pulse carrying $N$ photons with mean energy $\hbar \omega_{0}=$ $\hbar c k_{0}$, energy spread $\sigma=\hbar c \delta k$, and aperture $\alpha$. It applies for induced dipole transitions governed by the giant dipole resonance, i.e., for photon energies well within the interval $\left[E_{d}-E_{i}-2 \Gamma^{\downarrow}, E_{d}-E_{i}+2 \Gamma^{\downarrow}\right]$ where $\Gamma^{\downarrow} \gg \sigma$ is the spreading width. Furthermore, expression (30) holds for times $T \gg \hbar / \Gamma^{\downarrow}$.

\section{DISCUSSION}

\section{A. Parameter dependence of the rate}

The rate (30) has all the features that characterize a dipole transition for a stationary driving field: It is independent of time, it is proportional to the fine structure constant, to the third power of the transition energy, and to the square of the nuclear transition matrix element (the Lorentzian guarantees evaluation at the correct energy). Less obviously but not unexpectedly, the rate is proportional to the aperture of the pulse and to the total number of photons in the pulse. Combining the factor $\hbar c \delta k=\sigma$ with the Lorentzian and approximating the latter by $1 / \Gamma^{\downarrow}$, we interpret the ratio $\sigma / \Gamma^{\downarrow}$ as the fraction of the total energy range of the GDR available for dipole transitions that is actually illuminated by the width $\sigma$ of the laser pulse.

In quantum optics $[18,19]$ the rate is often written as the product of three factors: The fine structure constant, the square of the transition matrix element, and the energy density of the laser at the position of the atom. Equation (30) can also be written in that way. Indeed, aside from a factor $(2 \pi)^{3}$, the factor in big square brackets can be read as the energy density of the laser pulse at the position of the nucleus. The total energy of the pulse is $N \hbar \omega_{0}$, the first factor in big square brackets. The second factor in the big square brackets $\alpha k_{0}^{2} \delta k$ can be read as the inverse of the effective volume $V_{\text {eff }}$ of the pulse. From a formal point of view that is plausible because this factor is equal to $(2 \pi)^{3} \sum_{\vec{k}, \lambda}\left(1 / L^{3}\right)$; see Eqs. (28) and (29). The identification of the factor with the effective volume $V_{\text {eff }}$ of the laser pulse is made physically plausible as follows. Equation (2) shows that at $\vec{r}=0$ (the location of the target nucleus), the spatial parts of all modes $(\vec{k}, \lambda)$ have value unity, the laser field has maximum intensity. We identify $V_{\text {eff }}$ with the volume of the region surrounding the point $\vec{r}=0$ where the laser intensity is not significantly reduced by destructive interference. At $\vec{r}=0$ the intensity remains maximal during the entire duration time $\tau$ of the pulse. The extension of $V_{\text {eff }}$ in the direction $\vec{k}_{0}$ of propagation is, therefore, given by $\tau / c=$ $1 / \delta k$. In the plane perpendicular to $\vec{k}_{0}$, the minimum distance from the point $\vec{r}=0$ for destructive interference to become effective is given by the wave length $\lambda_{0}=(2 \pi) / k_{0}$. The area of the resulting circle centered at $\vec{r}=0$ is bounded from below by $2 \pi \lambda_{0}^{2}=(2 \pi)^{3} / k_{0}^{2}$. Such destructive interference can happen only if sufficiently many modes perpendicular to $\vec{k}_{0}$ are available, i.e., for sufficiently large values of the aperture $\alpha$ of the pulse. Decreasing $\alpha$ reduces the set of such transverse modes and increases the area of the circle. For $\alpha \rightarrow 0$ the pulse consists of plane waves all traveling in the direction $\vec{k}_{0}$. Destructive interference in the direction perpendicular to $\vec{k}_{0}$ is impossible, the area of the circle diverges. For $\alpha \ll 1$ and to lowest order in $\alpha$, the area is, therefore, of order $(2 \pi)^{3} /\left(\alpha k_{0}^{2}\right)$, 
and $V_{\text {eff }} /(2 \pi)^{3}$ is of order $1 /\left(\alpha k_{0}^{2} \delta k\right)$. That confirms our identification of the second factor in big square brackets with $(2 \pi)^{3} / V_{\text {eff }}$ where $V_{\text {eff }}$ is the inverse (effective) volume of the laser pulse. Although we have not used the energy density in our derivation, that concept naturally emerges in the interpretation of our result.

\section{B. Numerical estimate}

We give an order-of-magnitude estimate of the dipole width $\Gamma_{\text {dip }}$, defined as the product of $\hbar$ and the rate (30). Grouping the factors appropriately for our discussion, we obtain

$$
\begin{aligned}
\Gamma_{\text {dip }}= & \left(\frac{4 \pi \alpha}{9 \pi} \frac{e^{2}}{\hbar c}\right) \frac{\Gamma^{\downarrow} \sigma}{\left(E_{i}+\hbar \omega_{0}-E_{d}\right)^{2}+(1 / 4)\left(\Gamma^{\downarrow}\right)^{2}} \\
& \times\left(k_{0}^{2} \sum_{J_{f}}\left|\left\langle i_{N} J_{i}|| r Y_{1}\left(\vec{k}_{0}|| \Omega\right) \mid d\left(i_{N}\right) J_{f}\right\rangle\right|^{2}\right) \\
& \times N \hbar \omega_{0} .
\end{aligned}
$$

In Eq. (31), all factors but the last one are dimensionless, and $\Gamma_{\text {dip }}$ has the dimension energy. For medium-weight (heavy) nuclei, the excitation energy $E_{d}-E_{i}$ of the GDR has values around $12 \mathrm{MeV}(8 \mathrm{MeV})$, respectively. Theoretical estimates of the spreading width $\Gamma^{\downarrow}$ [25-31] depend on nuclear-structure properties but lie in the range 4-8 MeV. As mentioned below Eq. (30) our derivation applies for photon energies well within an interval $I$ defined by $E_{d}-E_{i}-2 \Gamma^{\downarrow} \leqslant$ $\hbar \omega_{0} \leqslant E_{d}-E_{i}+2 \Gamma^{\downarrow}$.

Assuming $4 \pi \alpha$ to be of order $10^{-1}$, we find that the first factor in big round brackets is approximately given by $(1 / 4) \times 10^{-5}$ and is independent of photon energy. Within the interval $I$ the second factor changes little with photon energy (provided that $\sigma$ is independent of $\hbar \omega_{0}$ ) and is roughly given by $2 \sigma / \Gamma^{\downarrow} \approx 20 \mathrm{keV} /(5 \mathrm{MeV})=4 \times 10^{-3}$. For a single nucleon, the dipole matrix element is of order $R_{N}$ (the nuclear radius), and we have $k_{0} R_{N} \approx 10^{-1}$. With $A$ the mass number, with $R_{N} \propto A^{1 / 3}$, and for photon energies at the center of the GDR, the product $k_{0} R_{N}$ changes by the factor $\left[(200)^{1 / 3} \times\right.$ $8] /\left[(100)^{1 / 3} \times 12\right] \approx 0.9$ as $A$ changes from medium-weight to heavy nuclei. It also changes little for values of $\hbar \omega_{0}$ within the interval $I$. As mentioned below Eq. (16) we have so far suppressed in our notation the sum over the contributions of neutrons and protons with effective charges for each type of nucleon. According to the dipole sum rule [32] (exhausted by the GDR if the sum over $J_{f}$ is included) that sum yields the factor $N Z / A$ where $Z(N)$ is the number of protons (neutrons), respectively, in the target nucleus, and where $A=Z+N$. We have $N Z / A \approx 25$ (50) for $A=100$ ( $A=200$, respectively). The second line in Eq. (31) is, thus, of order unity and depends weakly on photon energy. The combination of these factors yields $10^{-8}$. That factor is weakly dependent on energy, mainly via the Lorentzian in the first line of Eq. (31).

We conclude that the dipole width is of order $10^{-8} N \hbar \omega_{0}$. To significantly induce nuclear dipole transitions, the dipole width must lie in the $\mathrm{MeV}$ range. That value is attained for $N \approx 10^{8}$. In other words, for inducing significant dipole transitions it suffices that the pulse contains about $10^{8}$ photons- for backscattered photons a small fraction of the photon number in the original pulse prior to backscattering!

In the derivation we have assumed that $\hbar / \Gamma^{\downarrow}$ defines the smallest time scale in the dipole absorption process. That is the case as long as $\Gamma_{\text {dip }}<\Gamma^{\downarrow}$. Otherwise, nuclear equilibration itself must be included in the time-dependent description of dipole absorption. That would also require a modification of the rate equations describing the laser-nucleus interaction.

\section{Influence of finite nuclear lifetime}

How is the result (30) influenced by the finite lifetimes of the excited nuclear states? We focus attention on the state $f_{N}$. The time evolution of $f_{N}$ carries the factor $\exp \{-\gamma t\}$. Here $\gamma$ is twice the total width for spontaneous gamma decay in units of $\hbar$ [not to be confused with the spreading width introduced in Eq. (22)]. We accordingly replace in Eq. (16) the factor $\exp \left\{i\left(\omega_{k}-\omega_{f i}\right)\left(t^{\prime}-t\right)\right\}$ by $\exp \left\{i\left(\omega_{k}-\omega_{f i}\right)\left(t^{\prime}-\right.\right.$ $t)\} \exp \left\{-\left(t+t^{\prime}\right) \gamma\right\}$. Integrating that expression over $t$ and $t^{\prime}$ from zero to $T$ gives

$$
\begin{gathered}
\frac{1}{\left(\omega_{k}-\omega_{f i}\right)^{2}+\gamma^{2}}(1+\exp \{-2 \gamma T\} \\
\left.-2 \exp \{-\gamma T\} \cos \left[\left(\omega_{k}-\omega_{f i}\right) T\right]\right) .
\end{gathered}
$$

That expression replaces the last line in Eq. (20). It is obvious that we must require $\gamma T \ll 1$ as otherwise the excited state will undergo decay before the excitation process terminates. Then expression (32) reduces to the last line of Eq. (20), and we recover the result (30). The time for laser-nucleus interaction is bounded by $T \leqslant \hbar / \sigma$. For nuclear decay to be unimportant we must have $\hbar / \sigma \ll \gamma$. That constraint may not be strictly fulfilled in practice.

\section{STATIONARITY}

The derivation of Eq. (30) for the rate $R$ is based upon the stationarity condition (11). We identify the conditions of validity of that equation. As mentioned at the end of Sec. V, use of the stationarity condition necessarily implies a summation over photon states, in Sec. VII referred to as the sum over final photon states. We display the origin of that necessity and show that the sum extends over the states occupied in the incident laser pulse and is, therefore, actually a sum over initial photon states.

First, we simplify the presentation by considering the onedimensional case. We are well aware, of course, of the fact that this does not do justice to the case of electromagnetic waves. We continue to speak of the quanta in one dimension as of photons. We deviate from Sec. IV and begin with a laser pulse carrying a single photon only. In analogy to Eq. (5) we write the density matrix as

$$
\rho_{1}=\sum_{k l} \rho_{k l}|k, 1\rangle\langle l, 1| .
$$

In space representation, the single-photon state $|k, 1\rangle$ is a normalized plane wave $(1 / \sqrt{L}) \exp \{i k x)\}$ with quantized values of $k=2 \pi m / L$ and integer $m$. In second (field) quantization, the state $|k, 1\rangle=a_{k}^{\dagger}|0\rangle$ is obtained by applying the photon creation operator $a_{k}^{\dagger}$ to the vacuum state 
$|0\rangle$. It is instructive to have a model for the elements $\rho_{k l}$ of the density matrix. For the wave function $\psi(x)=$ $(1 / \sqrt{2 \pi d}) \exp \left\{i k_{0} x\right\} \exp \left\{-x^{2} /\left(2 d^{2}\right)\right\}$ of the laser pulse we take a normalized Gaussian of width $d$ and mean momentum $k_{0}$. In space representation the density matrix is $|\psi(x)\rangle\langle\psi(x)|$. In the representation (33) the elements $\rho_{k l}$ of the density matrix factorize and for large normalization volume $L \gg d$ are given by

$$
\rho_{k l}=\rho_{k} \rho_{l}^{*} \text { with } \rho_{k}=(2 d / L)^{1 / 2} \exp \left\{-\left(k-k_{0}\right)^{2} d^{2} / 2\right\} .
$$

In the continuum limit these obey $\sum_{k}\left|\rho_{k}\right|^{2}=1$. We calculate the total contribution of the $k$-dependent terms to Eq. (20) including the normalization factor without using the stationarity condition. The contribution consists of the terms analogous to the left-hand side of Eq. (11) and the terms proportional to $\rho_{1}^{(1)}$. We use $\operatorname{Tr}\left[\rho_{1} a_{k}^{\dagger} a_{l}\right]=\rho_{k} \rho_{l}^{*}=n_{k l}$. We follow Secs. IV and $\mathrm{V}$. The total contribution to the transition probability is

$$
\frac{1}{L}\left|\sum_{k} \sqrt{\omega_{k}} \rho_{k} \exp \left\{i\left(\omega_{k}-\omega_{f i}\right) T / 2\right\} \frac{\sin \left[\left(\omega_{k}-\omega_{f i}\right) T / 2\right]}{\omega_{k}-\omega_{f i}}\right|^{2} .
$$

By construction of the laser pulse, the summation over $k$ is confined to values of $\omega_{k}$ in an interval of width $\sigma / \hbar$ centered at $\omega_{0}$, the mean frequency.

To display the consequence of the stationarity condition (11), we write expression (35) as a double sum over $k$ and $l$. Use of the stationarity condition (11) reduces that double sum to a single sum over $k$ and yields

$$
\frac{1}{L} \sum_{k} \omega_{k} n_{k} \frac{\sin ^{2}\left[\left(\omega_{k}-\omega_{f i}\right) T / 2\right]}{\left(\omega_{k}-\omega_{f i}\right)^{2}},
$$

with the notation $n_{k}=\left|\rho_{k}\right|^{2}$. That expression is analogous to the right-hand side of Eq. (20). As in Sec. VII, the factor $1 / L$ is eventually removed by an additional summation over final states followed by $(1 / L) \sum_{k^{\prime}} \rightarrow(1 / 2 \pi) \int d k^{\prime}$.

To show how that prescription comes about, we write the modulus squared in expression (35) as a double sum over $k$ and $l$ and consider $T \gg \hbar / \sigma$. The arguments of both exponentials nearly cancel, the arguments of the two sin functions are almost identical, and so are the values of $\sqrt{\omega_{k}}$ and of $\sqrt{\omega_{l}}$. Moreover, Eq. (34) shows that in the considered frequency interval, $\rho_{l}$ changes very little, and we may put $\rho_{k} \rho_{l}^{*} \approx\left|\rho_{k}\right|^{2}=n_{k}$. Taking all that together expression (35) becomes equal to

$$
\sum_{k} \omega_{k} n_{k} \frac{\sin ^{2}\left[\left(\omega_{k}-\omega_{f i}\right) T / 2\right]}{\left(\omega_{k}-\omega_{f i}\right)^{2}} \frac{1}{L} \sum_{l} .
$$

The summation over $l$ is restricted to $\omega_{l}$ values that lie in the above-mentioned frequency interval, i.e., it extends over the states occupied in the laser pulse. With $\sum_{l} \rightarrow[L /(2 \pi)] \int d k^{\prime}$ we retrieve the result (36) obtained from the stationarity condition provided that the latter is augmented by the summation over final states. In hindsight it is obvious that the Kronecker delta in condition (11) does not arise naturally in the quasicontinuous description appropriate for large $L$ values. The Kronecker delta must be supplemented by the summation over states occupied in the primary laser pulse.
This argument can straightforwardly be generalized to the three-dimensional case and to the laser pulse (5) involving $N$ photons. In Sec. IV, the normalized states $|i, N\rangle=$ $\left|n_{1}^{(i)}\right\rangle\left|n_{2}^{(i)}\right\rangle \times \cdots \times\left|n_{M}^{(i)}\right\rangle$ are used to define in Eq. (5) the density matrix $\rho=\sum_{i j} \rho_{i j}|i, N\rangle\langle j, N|$. Introducing the notation $\rho_{N, l m}=\operatorname{Tr}\left[a_{l}^{\dagger} a_{m} \rho_{N}\right]$, this expression differs from zero only if $|j, N\rangle=a_{l}^{\dagger} a_{m}|i, N\rangle$. We denote that special state by $i(l, m)$. Thus,

$$
\operatorname{Tr}\left[a_{l}^{\dagger} a_{m} \rho_{N}\right]=\rho_{N, l m}=\sum_{i} \rho_{i, i(l, m)} .
$$

Obviously, $\sum_{l} \rho_{N, l l}=N$. The factor analogous to expression (35) becomes

$$
\begin{aligned}
& \frac{1}{L^{3}} \sum_{l m} \sqrt{\omega_{l}} \sqrt{\omega_{m}} \rho_{N, l m} \exp \left\{i\left(\omega_{l}-\omega_{f i}\right) T / 2\right\} \\
& \times \exp \left\{-i\left(\omega_{m}-\omega_{f i}\right) T / 2\right\} \frac{\sin \left[\left(\omega_{l}-\omega_{f i}\right) T / 2\right]}{\omega_{l}-\omega_{f i}} \\
& \quad \times \frac{\sin \left[\left(\omega_{l}-\omega_{f i}\right) T / 2\right]}{\omega_{l}-\omega_{f i}} .
\end{aligned}
$$

The arguments now are parallel to the ones for the onedimensional case. For large $T$ the sums in expression (39) are confined to values specified by the longitudinal and the lateral extension of the laser pulse in $k$ space, respectively. For fixed $l$ the dependence of the factor $\rho_{N, l m}$ on $m$ is smooth because $\rho_{N, l m}$ is effectively the element of a one-photon (not an $\mathrm{N}$ photon) density matrix. (In comparison to the one-photon case of Eq. (34), the summation over the states $i$ can only increase the smoothness of $\left.\rho_{N, l m}\right)$. For the same reason the sum over final states is a sum over single photon (and not $N$-photon) states. Thus, expression (39) becomes

$$
\sum_{l} \omega_{l} \rho_{l l} \frac{\sin ^{2}\left[\left(\omega_{l}-\omega_{f i}\right) T / 2\right]}{\left(\omega_{l}-\omega_{f i}\right)^{2}} \frac{1}{L^{3}} \sum_{k_{x}, k_{y}, k_{z}} .
$$

With $\rho_{l l}=n_{l}$ that is exactly the expression used in Eq. (20), were the latter augmented, however, by the summation over final states. These extend over the photon states occupied by the laser pulse.

\section{COHERENCE}

We have stated above that our result (30) for the dipole absorption rate is independent of the state of coherence of the pulse. For clarity we here define coherence as used in this paper. Doing so is perhaps necessary because naively, one might argue that the matrix element of the photon annihilation operator for a transition from a single-mode quantum state carrying $N$ photons to another state carrying $N-1$ photons is proportional to $\sqrt{N}$, its square is proportional to $N$. The factor $N$ in Eq. (30) would, thus, seem to be tied to the pulse carrying only a single mode and, hence, being coherent.

We consider a set of $n$ orthonormal wave packets

$$
\left|\Psi^{(k)}\right\rangle=\sum_{i} \Phi_{i}^{(k)}|i, N\rangle
$$


with $k=1, \ldots, n$ and $\sum_{i} \Phi_{i}^{(k)} \Phi_{i}^{(l) *}=\delta_{k l}$. Each of these carries $N$ photons. Needless to say, the localization in space and time of each of the $n$ wave packets $\Psi^{(k)}$ should be very similar. We compare two density matrices $\rho_{1}$ and $\rho_{2}$ constructed from the set $\left\{\left|\Psi^{(k)}\right\rangle\right\}$ and defined by

$$
\begin{aligned}
& \rho_{1}=\sum_{k} \alpha_{k}\left|\Psi^{(k)}\right\rangle \sum_{l}\left\langle\Psi^{(l)}\right| \alpha_{l}^{*}, \\
& \rho_{2}=\sum_{k} \alpha_{k}\left|\Psi^{(k)}\right\rangle\left\langle\Psi^{(k)}\right| \alpha_{k}^{*} .
\end{aligned}
$$

The $n$ complex coefficients $\alpha_{k}$ obey $\sum_{k}\left|\alpha_{k}\right|^{2}=1$. Both density matrices obey $\operatorname{Tr}(\rho)=1, \operatorname{Tr}\left(\sum_{k} a_{k}^{\dagger} a_{k} \rho\right)=N$, and $\rho=$ $\rho^{\dagger}$. The matrix elements $\left\langle\Psi_{f}|\mathcal{O}| \rho|\mathcal{O}| \Psi_{f}\right\rangle$ of these two density matrices, taken of an operator $\mathcal{O}$ with respect to some final state $\left|\Psi_{f}\right\rangle$, are

$$
\begin{aligned}
& \left\langle\Psi_{f}|\mathcal{O}| \rho_{1}|\mathcal{O}| \Psi_{f}\right\rangle=\sum_{k, l} \alpha_{k} \alpha_{l}^{*}\left\langle\Psi_{f}|\mathcal{O}| \Psi^{(k)}\right\rangle\left\langle\Psi^{(l)}|\mathcal{O}| \Psi_{f}\right\rangle \\
& \left\langle\Psi_{f}|\mathcal{O}| \rho_{2}|\mathcal{O}| \Psi_{f}\right\rangle=\sum_{k}\left|\alpha_{k}\right|^{2}\left|\left\langle\Psi_{f}|\mathcal{O}| \Psi^{(k)}\right\rangle\right|^{2}
\end{aligned}
$$

In Eq. (43) the matrix elements of $\rho_{1}$ allow for interference of the states $\left|\Psi^{(k)}\right\rangle$ and $\left|\Psi^{(l)}\right\rangle$ with $k \neq l$. Such interference terms are absent in the matrix elements of $\rho_{2}$ where only intensities are added. Therefore, the density matrix $\rho_{1}$ is said to be coherent, while $\rho_{2}$ is incoherent. That usage of the term coherence is completely consistent with Glauber's [17]. [The difference is that, instead of the basis states $\left|\Psi^{(k)}\right\rangle$, Glauber uses coherent states. But his distinction between a coherent density matrix (leading to interference fringes) in Eq. (2.322) and an incoherent one in Eq. (2.289) of Ref. [17] corresponds exactly to our distinction between $\rho_{1}$ and $\rho_{2}$, respectively, in our Eq. (42)].

A coherent (incoherent) laser pulse has a density matrix of the form $\rho_{1}\left(\rho_{2}\right.$, respectively). Suppose we repeat the calculation in previous sections for these two pulses, each carrying $N$ photons. In the first case we obtain directly the result (30). In the second case, each term in the sum over $k$ in Eq. (42) yields for the rate the result (30), multiplied by $\left|\alpha_{k}\right|^{2}$. The sum over $k$ then yields Eq. (30). That shows very clearly that the factor $N$ in the rate (30) is not due to coherence but results from the presence of $N$ photons in the pulse. We conclude that the rates for a completely coherent and for an incoherent laser pulse are the same. Coherence is not a necessary requirement for the boost factor $N$ to appear in Eq. (30) for the rate.

\section{SHORT TIMES}

In Ref. [5], the rate $R$ is used to calculate sequential multiple dipole absorption during the action of a single laser pulse. The difference in time between these absorption processes is of the order of $\hbar / \Gamma^{\downarrow}$ and, thus, about two orders of magnitude smaller than $\hbar / \sigma$. The form of $R$ derived in Eq. (30) for large times does not obviously apply in that case. We now show that, except for numerical factors of order unity, the rate (30) does indeed apply also for times $T \approx 2 \pi \hbar / \Gamma^{\downarrow}$, irrespective of the degree of coherence of the laser pulse. These are the times relevant for use of $R$ in rate equations.

We use Eq. (39) and confine ourselves to the relevant factors in Eq. (22). That gives

$$
\begin{aligned}
& \frac{N}{L^{3}} \int_{E_{d}-2 \Gamma^{\downarrow}}^{E_{d}+2 \Gamma^{\downarrow}} d E_{f} \frac{\Gamma^{\downarrow}}{2 \pi\left[\left(E_{f}-E_{d}\right)^{2}+(1 / 4)\left(\Gamma^{\downarrow}\right)^{2}\right]} \sum_{k l} \rho_{k l} \\
& \quad \times\left(2 \sqrt{\omega_{k}} \frac{\sin \left[\left(\omega_{k}-\omega_{f i}\right) T / 2\right]}{\left(\omega_{k}-\omega_{f i}\right)} \exp \left\{i\left(\omega_{k}-\omega_{f i}\right) T / 2\right\}\right) \\
& \quad \times\left(2 \sqrt{\omega_{l}} \frac{\sin \left[\left(\omega_{l}-\omega_{f i}\right) T / 2\right]}{\left(\omega_{l}-\omega_{f i}\right)} \exp \left\{-i\left(\omega_{l}-\omega_{f i}\right) T / 2\right\}\right) .
\end{aligned}
$$

With $\omega_{k}=\omega_{0}+\delta \omega_{k}$, the frequency increment $\delta \omega_{k}$ in the sum over $k$ ranges over an interval of size $\sigma / \hbar \ll \omega_{0}$ and analogously the same holds for $\omega_{l}$. Thus, $\sqrt{\omega_{k}} \approx \sqrt{\omega_{0}} \approx \sqrt{\omega_{l}}$. For $T \approx 2 \pi \hbar / \Gamma^{\downarrow}$, the ranges of $\delta \omega_{k} T \approx 2 \pi \sigma / \Gamma^{\downarrow}$ and of $\delta \omega_{l} T \approx$ $2 \pi \sigma / \Gamma^{\downarrow}$ are very small compared to unity. Therefore, we neglect $\delta \omega_{k}$ and $\delta \omega_{l}$ in the exponentials and in the Bessel functions. We obtain

$$
\begin{aligned}
& \frac{N T^{2} \omega_{0}}{L^{3}} \int_{E_{d}-2 \Gamma^{\downarrow}}^{E_{d}+2 \Gamma^{\downarrow}} d E_{f} \frac{\Gamma^{\downarrow}}{2 \pi\left[\left(E_{f}-E_{d}\right)^{2}+(1 / 4)\left(\Gamma^{\downarrow}\right)^{2}\right]} \\
& \quad \times\left(\frac{\sin \left[\left(\omega_{0}-\omega_{f i}\right) T / 2\right]}{\left(\omega_{0}-\omega_{f i}\right)(T / 2)}\right)^{2} \sum_{k l} \rho_{k l} .
\end{aligned}
$$

We estimate the last double sum by assuming that the wave packet describing the laser pulse is a product of three normalized Gaussians with widths $d_{l}, d_{\perp}, d_{\perp}$ in the longitudinal $(z)$ and in the transverse $(x, y)$ directions, respectively. Then $\rho_{k l}$ factorizes as in Eq. (34), with each factor $\rho_{k}$ given by $\left(8 d_{l} d_{\perp}^{2} / L^{3}\right)^{1 / 2} \exp \left\{-\left(k_{z}-k_{0}\right)^{2} d_{l}^{2}\right\} \exp \left\{-\left(k_{x}^{2}+\right.\right.$ $\left.\left.k_{y}^{2}\right) d_{\perp}^{2}\right\}$. The double sum becomes $\sum_{k l} \rho_{k l}=L^{3} /\left(\pi^{3} d_{l} d_{\perp}^{2}\right)$. That cancels the factor $L^{-3}$ in Eq. (45).

As done before we assume that $\omega_{0}$ is located in an interval centered at $E_{d}-E_{i}$ of width $\Gamma^{\downarrow}$. The Bessel function depends on the integration variable $E_{f}$ via $\omega_{f i}$. For times $T \leqslant \hbar / \Gamma^{\downarrow}$ the square of the Bessel function has a broad distribution which overlaps the range of the Lorentzian. The entire integral in Eq. (45) is then of order unity. The transition probability grows roughly quadratically with the time $T$. It is not possible then to give a meaningful definition of the rate. For increasing $T$ and $T>\hbar / \Gamma^{\downarrow}$ the width of the Bessel function becomes narrower than that of the Lorentzian. Eventually it is legitimate to pull the Lorentzian factor ahead of the integration, with $E_{f}$ replaced by $\omega_{0}+E_{i}$. Then one of the factors $T$ in expression (45) is absorbed by replacing $d E_{f} \rightarrow d\left(T E_{f} / \hbar\right)$, the time dependence of the transition probability becomes linear, and it is meaningful to define a rate. The transition between the two regimes is obviously smooth but happens around $T=2 \pi \hbar / \Gamma^{\downarrow}$. It is, thus, meaningful to evaluate the short-time rate $R^{\text {short }}$ at time $T=2 \pi \hbar / \Gamma^{\downarrow}$. It is given by

$$
\begin{aligned}
R^{\text {short }}= & c \frac{8 \pi^{2}}{3^{2}} \frac{e^{2}}{\hbar c}\left|\left\langle i_{N} J_{i}|| r Y_{1}\left(\vec{k}_{0}|| \Omega\right) \mid d\left(i_{N}\right) J_{f}\right\rangle\right|^{2} \\
& \times \frac{\Gamma^{\downarrow}}{\left(E_{i}+\hbar \omega_{0}-E_{d}\right)^{2}+(1 / 4)\left(\Gamma^{\downarrow}\right)^{2}}\left[\frac{N \hbar \omega_{0}}{\pi^{3} d_{0} d_{\perp}^{2}}\right] .
\end{aligned}
$$


We compare that result with expression (30) for $R$ obtained for $T \gg \hbar / \Gamma^{\downarrow}$. Since $\delta k_{0}$ is inversely proportional to the length of the laser pulse and $\alpha k_{0}^{2}$ is inversely proportional to the area in the lateral direction, the two expressions agree except for a numerical factor of order unity. We conclude that - except for a factor of order unity - the full rate (30) is attained already at short times of order $2 \pi \hbar / \Gamma^{\downarrow}$. The use of that expression in nuclear rate equations is, thus, fully justified. It makes sense that the rate cannot be meaningfully defined for times smaller than $\hbar / \Gamma^{\downarrow}$ because that time marks the end of the equilibration process following dipole absorption. Likewise it is not surprising that after equilibration the rate changes little up to very large times.

We note that in the present section, it was necessary to use explicitly an assumption on the shape of the laser pulse. No such assumption was needed in the derivation of Sec. IX, which makes use only of general properties (energy spread and aperture) of the pulse. We also note that Eq. (46) holds irrespective of the coherence properties of the laser pulse.

\section{COMPARISON WITH ATOMIC PHYSICS}

A comparison with the treatment of laser-induced photon absorption processes in atoms reveals striking and illuminating differences. In atoms the relevant photon energies are of order $\mathrm{eV}$, and the product of wave number $k$ and atomic radius $R$ also obeys $k R \ll 1$, justifying the use of the dipole approximation. In that approximation and in the interaction picture the Hamiltonian is customarily written as [18]

$$
\mathcal{H}_{\text {int }}=-e \vec{q}(t) \cdot \vec{E}_{\mathrm{op}}(\vec{r}, t)
$$

where $\vec{E}_{\text {op }}$ denotes the operator of the electric field strength, taken at the position $\vec{r}$ of the atomic nucleus and for times $t$ defined by the presence of the laser pulse, while $\vec{q}$ denotes the sum of the position operators of the electrons relative to the atomic nucleus. It is easily seen that in dipole approximation the forms (1) and (47) for the Hamiltonian are equivalent.

An approximation commonly used in atomic physics replaces the operator $\vec{E}_{\text {op }}$ by the classical field strength $\vec{E}$. [The expectation value of $\vec{E}_{\text {op }}$ for the density matrix of Sec. IV actually vanishes, and the classical field strength $\vec{E}$ must be defined via the equality of $(\vec{E})^{2}$ and the expectation value of $\left(\vec{E}_{\mathrm{op}}\right)^{2}$.] That approximation has the great advantage that the action of the classical field on the atomic electrons can be followed beyond ionization threshold [33]. Why don't we adopt the same approach for nuclei? Scaling arguments yield the answer. In the scenario of the flying mirror, scaling is due to the factor $\eta=4 \gamma_{e}^{2}$ that describes backscattering of the incident laser pulse. (A similar relativistic boost factor is involved in production of $\mathrm{MeV}$ photons at the Gamma Factories.) Both the mean photon energy $\hbar \omega$ and the energy spread prior to backscattering are multiplied by $\eta$, the pulse length $l$ in the direction of propagation is multiplied by $\eta^{-1}$. We assume that in the radial direction the width $r$ of the pulse remains unchanged. Thus the volume $V=2 \pi l r^{2}$ of the pulse is scaled by the factor $\eta^{-1}$. The scaling of the classical field strength follows from Poynting's theorem $(e E)^{2}=N_{0} \hbar \omega /(2 V)$. Under the unrealistic assumption that all $N_{0} \approx 10^{11}$ photons in the incident pulse are backscattered, $E^{2}$ is scaled by the factor $\eta^{2}$ and the field strength itself by the factor $\eta$. With $\eta \approx 10^{6}-10^{7}$ that converts a realistic atomic field strength of $1 \mathrm{eV} / \AA$ into less than $10^{-4} \mathrm{MeV} / \mathrm{fm}$ for an almost planar backscattered wave with minimal aperture $\alpha \propto\left(\delta k / k_{0}\right)^{2}$. The value $10^{-4}$ $\mathrm{MeV} / \mathrm{fm}$ is much too small to cause substantial nuclear excitation. That is confirmed by explicit numerical simulations using a three-dimensional Hartree-Fock code [34].

In conclusion, the standard approach used in atomic physics would yield negligible excitation probabilities for nuclei. Nuclear equilibration comes to the rescue. It requires the use of rate equations and leads to multiple photon absorption processes. Thanks to the sum over final states, the rate for dipole excitation is substantial, even if only a minute fraction of the $N_{0}$ photons in the incident laser pulse is backscattered.

\section{SUMMARY}

At excitation energies of several $\mathrm{MeV}$ or more, mediumweight and heavy nuclei tend to equilibrate on very short-time scales of the order of $\hbar / \Gamma^{\downarrow}$. Here $\Gamma^{\downarrow} \approx 5 \mathrm{MeV}$ is the spreading width of the nuclear GDR and accounts for equilibration after dipole excitation. Due to this fast equilibration, a nuclear reaction induced by a laser pulse carrying $N$ photons of mean energy $\hbar \omega_{0} \approx 5 \mathrm{MeV}$ or more should be described in terms of rate equations, in striking contrast to the laser-atom interaction which is often described in terms of a standard Hamiltonian involving the (classical) electromagnetic field strength. In the nuclear context, that approach would yield negligibly small excitation probabilities. Rate equations are the only viable alternative. Such equations use the rate $R$ for laser-induced nuclear dipole excitation as input. For photons with energies in the 5-10-MeV range the dipole approximation is appropriate. We have used the Brink-Axel hypothesis: A GDR exists as a viable mode of excitation not only for the nuclear ground state but also for every excited state of the nucleus. The expression derived for $R$ and given in Eq. (30) constitutes the central result of the paper. We have used the long-time limit $T \gg \hbar / \Gamma^{\downarrow}$, and the ensuing stationarity condition on the density matrix of the laser pulse. The rate is proportional to $\left(\hbar \omega_{0}\right)^{3}$ and to $N$. The factor $N$ applies independently of the degree of coherence of the laser pulse.

We have extensively discussed the physical interpretation of the rate $R$ and its dependence on laser and nuclear parameters. Examining the stationarity condition, we have shown why that condition has to be supplemented by a summation over final states. We have shown that, except for factors of order unity, expression (30) for $R$ holds also for short times of the order $2 \pi \hbar / \Gamma^{\downarrow}$. That is a sensible result because nuclear equilibration essentially terminates at that time. In consequence the values of the rate at time $2 \pi \hbar / \Gamma^{\downarrow}$ and at very large time $T \geqslant \hbar / \sigma$ differ only by a numerical factor of order unity. It follows that estimates based upon expression (30) can reliably be used in nuclear rate equations simulating multiple photon absorption from a single laser pulse. Equally important, Eq. (30) should help in optimizing laser pulses for the experimental investigation of such processes. An important element is the dependence of $R$ on the aperture $\alpha$ of the 
incident pulse. For the flying mirror, coherent backscattering is not required, and only a small fraction of the photons in the secondary laser pulse need be Compton backscattered. Conversely, experimental results on the laser-nucleus interaction would provide a test of the basic assumptions that underlie our approach, and on the results obtained. These are the Brink-Axel hypothesis for highly excited nuclear states and, in the calculations reported in Refs. [4,5], the values of the nuclear level density.

\section{ACKNOWLEDGMENT}

This work was part of and supported by the DFG Collaborative Research Center "SFB 1225 (ISOQUANT)."
[1] Extreme Light Infrastructure Nuclear Physics (ELI-NP), Official Website (2019), https://www.eli-np.ro/.

[2] W. Płaczek, A. Abramov, S. E. Alden, R. Alemany Fernandez, P. S. Antsiferov, A. Apyan, H. Bartosik, E. G. Bessonov, N. Biancacci, J. Bieron et al., Acta Phys. Pol. B 50, 1191 (2019).

[3] H. A. Weidenmüller, Phys. Rev. Lett. 106, 122502 (2011).

[4] A. Pálffy and H. A. Weidenmüller, Phys. Rev. Lett. 112, 192502 (2014).

[5] A. Pálffy, O. Buss, A. Hoefer, and H. A. Weidenmüller, Phys. Rev. C 92, 044619 (2015).

[6] D. Kiefer, M. Yeung, T. Dzelzainis, P. Foster, S. Rykovanov, C. Lewis, R. Marjoribanks, H. Ruhl, D. Habs, J. Schreiber et al., Nat. Commun. 4, 1763 (2013).

[7] A. Einstein, Ann. Phys. (Leipzig) 322, 891 (1905).

[8] T. Zh. Esirkepov, S. V. Bulanov, A. G. Zhidkov, A. S. Pirozhkov, and M. Kando, Eur. Phys. J. D 55, 457 (2009).

[9] D. Kiefer, A. Henig, D. Jung, D. C. Gautier, K. A. Flippo, S. A. Gaillard, S. Letzring, R. P. Johnson, R. C. Shah, T. Shimada et al., Eur. Phys. J. D 55, 427 (2009).

[10] J. Meyer-ter-Vehn and H.-C. Wu, Eur. Phys. J. D 55, 433 (2009).

[11] G. Mourou and T. Tajima, Science 331, 41 (2011).

[12] S. V. Bulanov, T. Z. Esirkepov, M. Kando, A. S. Pirozhkov, and N. N. Rosanov, Phys. Usp. 56, 429 (2013).

[13] J. Mu, F.-Y. Li, M. Zeng, M. Chen, Z.-M. Sheng, and J. Zhang, Appl. Phys. Lett. 103, 261114 (2013).

[14] F. Y. Li, Z. M. Sheng, M. Chen, H. C. Wu, Y. Liu, J. Meyer-terVehn, W. B. Mori, and J. Zhang, Appl. Phys. Lett. 105, 161102 (2014).

[15] P. Thirolf (private communication).

[16] P. Ring and P. Schuck, The Nuclear Many-Body Problem (Springer-Verlag, New York, 1980).

[17] R. J. Glauber, Quantum Theory of Optical Coherence (Wiley, Weinheim, 2007).
[18] M. O. Scully and M. S. Zubairy, Quantum Optics (Cambridge University Press, Cambridge, UK, 1997).

[19] R. Loudon, The Quantum Theory of Light (Oxford Science Publications, New York, 2009).

[20] A. J. F. Siegert, Phys. Rev. 52, 787 (1937).

[21] A. R. Edmonds, Angular Momentum in Quantum Mechanics (Princeton University Press, Princeton, 1996).

[22] E. Merzbacher, Quantum Mechanics (John Wiley and Sons, London, 1970).

[23] M. Brink, Ph.D. thesis, University of Oxford, Oxford, UK, 1955.

[24] P. Axel, Phys. Rev. 126, 671 (1962).

[25] J. A. Maruhn, P. G. Reinhard, P. D. Stevenson, J. R. Stone, and M. R. Strayer, Phys. Rev. C 71, 064328 (2005).

[26] W. Kleinig, V. O. Nesterenko, J. Kvasil, P.-G. Reinhard, and P. Vesely, Phys. Rev. C 78, 044313 (2008).

[27] F. Dönau, G. Rusev, R. Schwengner, A. R. Junghans, K. D. Schilling, and A. Wagner, Phys. Rev. C 76, 014317 (2007).

[28] K. Yoshida and N. V. Giai, Phys. Rev. C 78, 014305 (2008).

[29] S. Peru, G. Gosselin, M. Martini, M. Dupuis, S. Hilaire, and J.-C. Devaux, Phys. Rev. C 83, 014314 (2011).

[30] T. Oishi, M. Kortelainen, and N. Hinohara, Phys. Rev. C 93, 034329 (2016).

[31] A. P. Severyukhin, S. Aberg, N. N. Arsenyev, and R. G. Nazmitdinov, Phys. Rev. C 98, 044319 (2018).

[32] J. M. Blatt and V. F. Weisskopf, Theoretical Nuclear Physics (Springer-Verlag, New York, 1979).

[33] S. V. Popruzhenko, J. Phys. B: At., Mol. Opt. Phys. 47, 204001 (2014)

[34] J. A. Maruhn, P.-G. Reinhard, P. Stevenson, and S. Umar, Comput. Phys. Commun. 185, 2195 (2014). 